\title{
An Introduction to Sequential Pentuals
}

\author{
${\text { Yogesh } \text { Agrawal }^{1} \text {, Faisal Kaleem }}^{1} \&$ David Hare ${ }^{1}$ \\ ${ }^{1}$ University of Louisiana Monroe, Monroe, USA \\ ${ }^{2}$ Department of Chemistry, State University of New York at Binghamton, Vestal, NY, USA \\ Correspondence: Correspondence: Faisal Kaleem, University of Louisiana at Monroe, 700 University Ave, Monroe, LA \\ 71209, USA.
}

Received: August 19, 2020

Accepted: September 22, 2020 Online Published: September 29, 2020

doi:10.5539/ijsp.v9n6p49

URL: https://doi.org/10.5539/ijsp.v9n6p49

\begin{abstract}
In this paper we introduce the idea of a pentual which is a 5-player extension of the well-known idea of a duel. We find the elimination probabilities of players under the assumption that each player tries to eliminate the strongest survivor at every stage. We focus mainly on the winning probability of the strongest player among the five and show that in many plausible situations this player does not even have a $20 \%$ chance of winning the game.
\end{abstract}

Keywords: pentual, sequential, winning

\section{Introduction}

"Survival of the fittest," a term famous in Darwinism, tells us that competition for survival is common in daily life where ultimately the best survives. This theory is often put to test by the Mathematical field of Game Theory which studies and evaluates the chances of certain outcomes. A duel is a perfect illustration of this idea where two people take shots at each other until one of the players dies and the other survives. A 'Pentual' is an extended version of the duel, where five players compete against each other instead of two. They shoot at each other until only one of them survives. Assigning constant marksmanship abilities to the players throughout the game, we derive certain interesting results, concentrating mainly on how the strongest marksman would fare if the firing turns occur in the reverse order of marksmanship.

The most well-known extention of a duel is a truel where three people compete in a shooting contest and each one tries to adopt a strategy so as to maximize their chances of being the only survivor. The term truel was possibly first used by Shubik (1975). Different variations of truels have been examined by various people. For example, Random Truels, in which the next shooter is randomly selected (as opposed to everyone getting "equal" turns) are discussed by Amengual and Toral (2006). Simultaneous Truels, in which everyone fires at the same time, have been discussed by Kilgour (1971). Kilgour (1975) later discusses sequential truels in detail. A quite comprehensive overview of truels is provided by Kilgour and Brams (1997). According to this reference, the first mathematical problem/puzzle involving truels (without using the term) appeared in Encyclopedia of Puzzles and Pastimes (Kinnaird, 1946). Quite recently, Hare and Kaleem (2019) did some work on expected winning probabilities in sequential truels under uniform distributions.

In the current study we extend this idea to five contestants and hence the name pentual. Thus, pentual will be a game in which five players participate and try to eliminate each other. The lone survivor wins. Each of the players will generally have different shooting capacities, which will be quantified by the probability of success of their shot. We study the various possible outcomes under certain assumptions and aim to derive the formulas for winning probabilities of different players. We also display a set of shooting capabilities for which the null hypothesis doesn't hold, the null hypothesis being that the winning probability of a player is directly proportional to their shooting capabilities (the fittest survives as put forward by Darwinism).

We start by making some convenient and reasonable assumptions. First, we assume that the shooting probabilities of the players remain constant during the game and are not affected by factors like stamina because the game needs to continue indefinitely until a winner is found. Next, we assume perfect competition. A perfect competition is defined in economics as a competition where everyone has complete knowledge of everything and hence can act accordingly. So, in our game all the players will be aware of each other's shooting probabilities and strategy, and hence can target a player accordingly. We also assume that each player, at any point of the game, will attack the surviving player (other than themselves, of course) with the highest shooting probability. This strategy is obvious in case of a truel since everyone would like to eliminate the strongest player because this elimination will mean that they end up in a duel with 
the weaker player. After a few attempts, we were not able to come up with a rigorous proof of whether this should be the correct strategy in case of a pentual, but it seems highly plausible that every player will always prefer to go from a pentual to a "quadruel" with the weakest possible set of players, and hence would try to eliminate the strongest player in the pentual, other than themselves. Similarly, everyone who makes to the quadruel will wish to enter the truel with weakest possible set of players and hence will try to eliminate the strongest player in the quadruel. The reader should not be confused by the fact that in most real-life situations, in order to survive in a limited resource situation, we don't always want to eliminate our strongest competitor, but sometimes we are equally happy by eliminating some weaker ones. The reason for this is that we don't always have to be the best in a field in order to survive. However, in a pentual, it is no use being the $2^{\text {nd }}$ best because it is a winner takes all situation. Moreover, any competitors that are out there are trying to eliminate everyone else and sooner or later every person will be under attack. So, it seems the best strategy for everyone is to try to eliminate the best shot at every turn.

\section{Rules of the Game}

- Consider 5 players A, B, C, D and E whom we will also refer to as the $1^{\text {st }}, 2^{\text {nd }}, 3^{\text {rd }}, 4^{\text {th }}$, and $5^{\text {th }}$ players, respectively.

- Shooting capacities of players are in descending order implying that player A has the best shooting ability and player E has the worst shooting probability.

- Once a player hits their target, the target is assumed dead and eliminated.

- All players shoot in turn with player E taking first shot forming an of order E, D, C, B, A, and back again to E following the cycle and removing any eliminated player(s). This reverse order of marksmanship is an attempt to remove some of the advantage that a stronger shot has over a weaker one and hence make the game more interesting.

- Success of the shot don't alter the sequence of future shots. So, if C attacks A, then no matter whether A gets killed or not, the next turn goes to B.

- Every player has the freedom to shoot at any of the other players. So, say C does not have to necessarily shoot at B or D. C can shoot at A, B, D or E.

- Abstaining from the shot is not allowed. This means that a player can neither choose to skip their shot or simply shoot in the air knowingly. Abstaining has been found to be a useful technique in some of the scenarios of a truel as shown by Kilgour (1975) but for the simplification of our study, we remove the abstinence as an option.

- Only one player shoots at a time. Thus, we consider only a "Sequential Pentual" for the most part. This is different from a "Simultaneous Pentual" in which players have the option to fire all at the same time.

\section{The Basic Approach}

Let us now return to the main focus of this paper, namely, the Sequential Pentual. To determine the winning probability of a player, we will start by determining their elimination at every level probability. Since a player can only win if s/he is the only player surviving at the end. This means that the player should not be the first to get killed, should not be the second to get killed, should not be the third to get killed and should not be the fourth to get killed as well. Hence, we will calculate each players' chances of being killed first, second, third, or fourth. The sum of these four results will give the probability of that player being killed at some point during the game. The complement of this sum will thus give the winning probability of the player under consideration. Many of the techniques will be the same as used by Hare and Kaleem (2019). However, at some points, it will be seen that there is some added complexity in the pentual calculations.

The following flow chart shows how the events of game can take place. As everyone will attack A in the beginning, and A will always attack B as long as everyone is alive, the first person to die can only be A or B. 


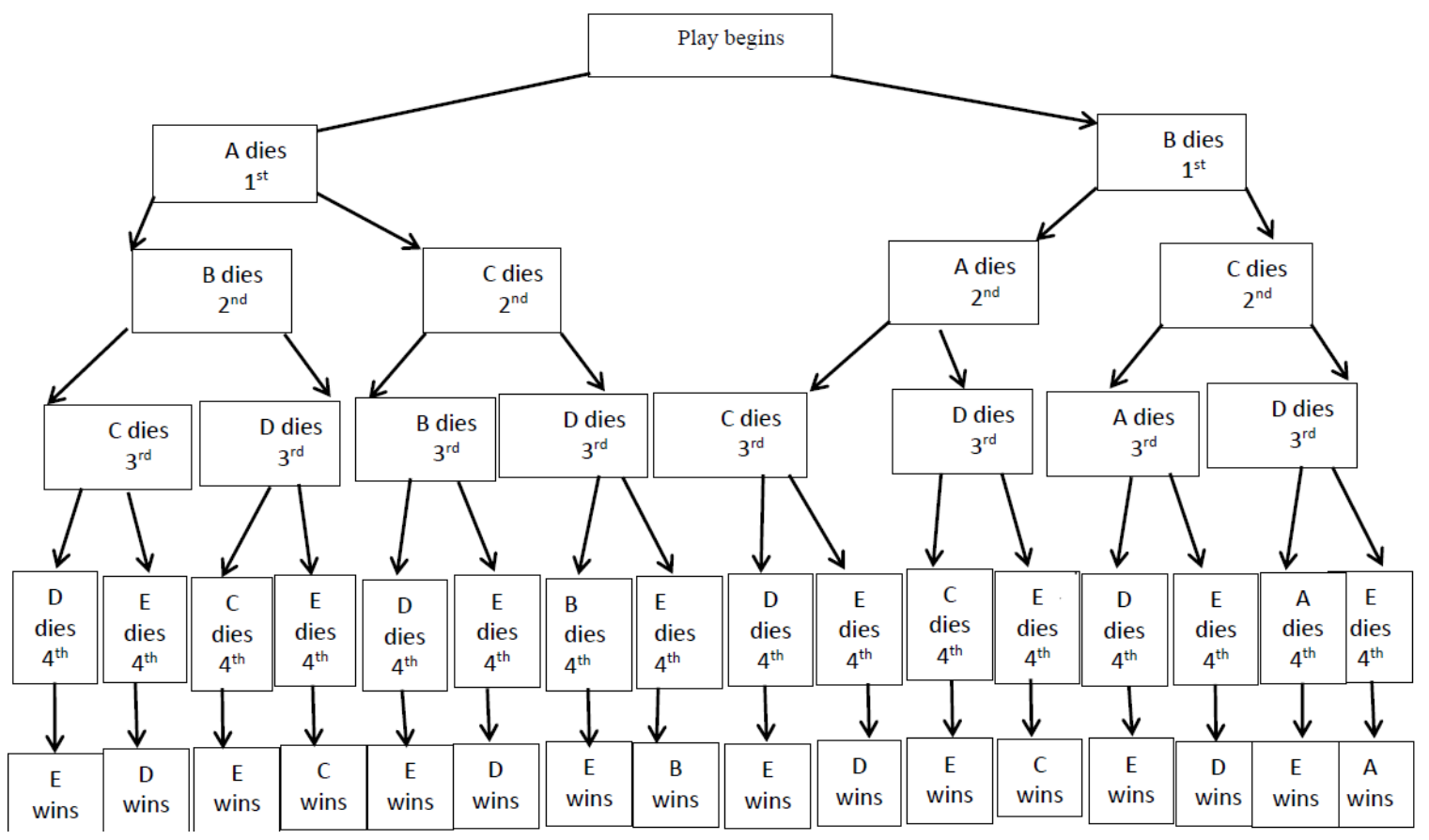

Figure 1. Flow of the Game

Similarly, the $2^{\text {nd }}$ player to die will be the A/B survivor or C, and so on. So, we can see that at the first level, only A or B can die. Continuing along various levels the eventual winner along each route is shown in the last row. In the calculations that follow, we indicate the shooting probabilities of $A, B, C, D, E$ by $a, b, c, d, e$, respectively. Also, we will denote complements by bars. Thus:

$$
\bar{a}:=1-a
$$

etc. Finally, the probability of the event that the $i^{\text {th }}$ player is the $j^{\text {th }}$ one to be eliminated, and is eliminated by the $k^{\text {th }}$ player will be denoted by $P_{i j k}$; while the probability of the event that the $i^{\text {th }}$ player is the $j^{\text {th }}$ one to be eliminated will be denoted by $P_{i j}$

\section{Calculation of $1^{\text {st }}$ Death Probabilities}

\section{(i) A is the First one to be Eliminated}

Since everyone will attack A, A can be killed by either E, D, C or B. If $\mathrm{E}$ is the one to eliminate A, we have:

$\mathrm{P}(\mathrm{E}$ kills $\mathrm{A})=\mathrm{P}\left(\mathrm{E}\right.$ kills $\mathrm{A}$ on $1^{\text {st }}$ try $)+\mathrm{P}\left(\mathrm{E}\right.$ kills $\mathrm{A}$ on $2^{\text {nd }}$ try $)+\ldots$

This is the same approach that was taken by Hare and Kaleem (2019) in a truel scenario. Thus, using our notation and the infinite sum formula for a geometric series we get:

$$
P_{115}=e+\bar{e} \bar{d} \bar{c} \bar{b} e+\cdots
$$

If $\mathrm{D}$ is the one to eliminate $\mathrm{A}$, we have by similar reasoning:

$$
P_{114}=\frac{\bar{e} d}{1-\bar{e} \bar{d} \bar{c} \bar{b} \bar{a}}
$$

Similarly, it can be seen that:

$$
\begin{aligned}
P_{113} & =\frac{\bar{e} \bar{d} c}{1-\bar{e} \bar{d} \bar{c} \bar{b} \bar{a}} \\
P_{112} & =\frac{\bar{e} \bar{d} \bar{c} b}{1-\bar{e} \bar{d} \bar{c} \bar{b} \bar{a}}
\end{aligned}
$$


Adding all these probabilities we see that:

$$
P_{11}=\frac{e+\bar{e} d+\bar{e} \bar{d} c+\bar{e} \bar{d} \bar{c} b}{1-\bar{e} \bar{d} \bar{c} \bar{b} \bar{a}}
$$

\section{(ii) B is the First one to be Eliminated}

Since only $\mathrm{A}$ is going to target $\mathrm{B}$, the calculations are much simpler in this case.

$\mathrm{P}(\mathrm{B}$ dies First $)=\mathrm{P}(\mathrm{A}$ kills $\mathrm{B})=\mathrm{P}\left(\mathrm{A}\right.$ kills $\mathrm{B}$ on $1^{\text {st }}$ try $)+\mathrm{P}\left(\mathrm{A}\right.$ kills $\mathrm{B}$ on $2^{\text {nd }}$ try $)+\ldots$

Using our notation, and the same approach as in case (i) we get:

\section{Calculation of $2^{\text {nd }}$ Death Probabilities}

$$
P_{21}=\frac{\bar{e} \bar{d} \bar{c} \bar{b} a}{1-\bar{e} \bar{d} \bar{c} \bar{b} \bar{a}}
$$

In this case, as discussed earlier, the 2 players who will be targeted will be $\mathrm{C}$ and the $\mathrm{A} / \mathrm{B}$ survivor. $\mathrm{C}, \mathrm{D}$, and $\mathrm{E}$ will target the A/B survivor, while the A/B survivor will target $\mathrm{C}$. One factor which adds to the complexity of calculations at this level is the fact that we arrive at this level through two possible routs: A being eliminated first, or B being eliminated first.

\section{Case I: B was the First one to Die}

This means A killed B. That means it is now E's turn to shoot, and the next person to die will be either A or C.

$\mathrm{P}\left(\mathrm{A}\right.$ dies $\left.2^{\text {nd }}\right)=\mathrm{P}(\mathrm{A}$ is killed by $\mathrm{E}$ or $\mathrm{D}$ or $\mathrm{C}) * \mathrm{P}\left(\mathrm{B} \operatorname{died} 1^{\text {st }}\right)$

Using our notation, we have:

$$
P_{12}=\left(P_{125}+P_{124}+P_{123}\right) * P_{21}
$$

Using the ideas and the result of section 6 we get:

Similarly,

$$
P_{12}=\left(\frac{e+\bar{e} d+\bar{e} \bar{d} c}{1-\bar{e} \bar{d} \bar{c} \bar{a}}\right) *\left(\frac{\bar{e} \bar{d} \bar{c} \bar{b} a}{1-\bar{e} \bar{d} \bar{c} \bar{b} \bar{a}}\right)
$$

$\mathrm{P}\left(\mathrm{C}\right.$ dies $2^{\text {nd }}$ given $\mathrm{A}$ killed $\left.\mathrm{B}\right)=\mathrm{P}(\mathrm{C}$ is killed by $\mathrm{A}) * \mathrm{P}\left(\mathrm{B} \operatorname{died} 1^{\text {st }}\right)$

\section{Case II: A was the First one to Die}

$$
=\left(\frac{\bar{e} \bar{d} \bar{c} a}{1-\bar{e} \bar{d} \bar{c} \bar{a}}\right) *\left(\frac{\bar{e} \bar{d} \bar{c} \bar{b} a}{1-\bar{e} \bar{d} \bar{c} \bar{b} \bar{a}}\right)
$$

It turns out that in this case we need to know who eliminated A because that will decide who gets the next shot, which is important to know in order to continue our calculations. If E killed A, then D has the next turn. So,

$\mathrm{P}\left(\mathrm{E}\right.$ killed $\mathrm{A}$, and $\mathrm{B}$ dies $\left.2^{\text {nd }}\right)=P_{115} *[\mathrm{P}(\mathrm{D}$ kills $\mathrm{B})+\mathrm{P}(\mathrm{C}$ kills $\mathrm{B})+\mathrm{P}(\mathrm{E}$ kills $\mathrm{B})]$

Similarly, we have:

$$
=\frac{e}{1-\overline{\mathrm{e}} \overline{\mathrm{d}} \overline{\mathrm{c}} \overline{\mathrm{b}} \overline{\mathrm{a}}} * \frac{d+\bar{d} c+\bar{d} \bar{c} \bar{b} e}{1-\bar{e} \bar{d} \bar{c} \bar{b}}
$$

$\mathrm{P}\left(\mathrm{E}\right.$ killed $\mathrm{A}$ and $\mathrm{C}$ dies $\left.2^{\text {nd }}\right)=P_{115} * \mathrm{P}(\mathrm{B}$ kills $\mathrm{C})$

$$
=\frac{e}{1-\overline{\mathrm{e}} \overline{\mathrm{d}} \overline{\mathrm{c}} \overline{\mathrm{b}} \overline{\mathrm{a}}} * \frac{\bar{d} \bar{c} b}{1-\bar{e} \bar{d} \bar{c} \bar{b}}
$$

Now if A was killed by $D$, then the next shot goes to $C$. Thus, in this case we will have:

$\mathrm{P}\left(\mathrm{D}\right.$ killed $\mathrm{A}$, and $\mathrm{B}$ dies $\left.2^{\text {nd }}\right)=P_{114} *[\mathrm{P}(\mathrm{E}$ kills $\mathrm{B})+\mathrm{P}(\mathrm{D}$ kills $\mathrm{B})+\mathrm{P}(\mathrm{C}$ kills $\mathrm{B})]$

$$
=\frac{\bar{e} d}{1-\bar{e} \bar{d} \bar{c} \bar{b} \bar{a}} * \frac{c+\bar{c} \bar{b} e+\bar{c} \bar{b} \bar{e} d}{1-\bar{e} \bar{d} \bar{c} \bar{b}}
$$


$\mathrm{P}\left(\mathrm{D}\right.$ killed $\mathrm{A}$, and $\mathrm{C}$ dies $\left.2^{\text {nd }}\right)=P_{114} * \mathrm{P}(\mathrm{B}$ kills $\mathrm{C})$

$$
=\frac{\bar{e} d}{1-\bar{e} \bar{d} \bar{c} \bar{b} \bar{a}} * \frac{\bar{c} b}{1-\bar{e} \bar{d} \bar{c} \bar{b}}
$$

If $\mathrm{A}$ was killed by $\mathrm{C}$, then the next shot goes to $\mathrm{B}$. In this case we will have:

$\mathrm{P}\left(\mathrm{C}\right.$ killed $\mathrm{A}$, and $\mathrm{B}$ dies $\left.2^{\text {nd }}\right)=P_{113} * \mathrm{P}[(\mathrm{E}$ kills $\mathrm{B})+\mathrm{P}(\mathrm{D}$ kills $\mathrm{B})+\mathrm{P}(\mathrm{C}$ kills $\mathrm{B})]$

$\mathrm{P}\left(\mathrm{C}\right.$ killed $\mathrm{A}$, and $\mathrm{C}$ dies $\left.2^{\text {nd }}\right)=P_{113} * \mathrm{P}(\mathrm{B}$ kills $\mathrm{C})$

$$
=\frac{\bar{e} \bar{d} c}{1-\bar{e} \bar{d} \bar{c} \bar{b} \bar{a}} * \frac{\bar{b} e+\bar{b} \bar{e} d+\bar{b} \bar{e} \bar{d} c}{1-\bar{e} \bar{d} \bar{c} \bar{b}}
$$

$$
=\frac{\bar{e} \bar{d} c}{1-\bar{e} \bar{d} \bar{c} \bar{b} \bar{a}} * \frac{b}{1-\bar{e} \bar{d} \bar{c} \bar{b}}
$$

If $\mathrm{A}$ was killed by $\mathrm{B}$, then the next shot goes to $\mathrm{E}$

$\mathrm{P}\left(\mathrm{B}\right.$ killed $\mathrm{A}$, and $\mathrm{B}$ dies $\left.2^{\text {nd }}\right)=P_{112} *[\mathrm{P}(\mathrm{E}$ kills $\mathrm{B})+\mathrm{P}(\mathrm{D}$ kills $\mathrm{B})+\mathrm{P}(\mathrm{C}$ kills $\mathrm{B})]$

$$
=\frac{\bar{e} \bar{d} \bar{c} b}{1-\bar{e} \bar{d} \bar{c} \bar{b} \bar{a}} * \frac{e+\bar{e} d+\bar{e} \bar{d} c}{1-\bar{e} \bar{d} \bar{c} \bar{b}}
$$

$\mathrm{P}\left(\mathrm{B}\right.$ killed $\mathrm{A}$, and $\mathrm{C}$ dies $\left.2^{\text {nd }}\right)=P_{112} * \mathrm{P}(\mathrm{B}$ kills $\mathrm{C})$

$$
=\frac{\bar{e} \bar{d} \bar{c} b}{1-\bar{e} \bar{d} \bar{c} \bar{b} \bar{a}} * \frac{\bar{e} \bar{d} \bar{c} b}{1-\bar{e} \bar{d} \bar{c} \bar{b}}
$$

Now we are in a position to find the probabilities of each player dying $2^{\text {nd }}$.

$\mathrm{P}\left(\mathrm{C}\right.$ dies $\left.2^{\text {nd }}\right)=\mathrm{P}\left(\mathrm{E}\right.$ killed $\mathrm{A}$, and $\left.\mathrm{C} \operatorname{dies} 2^{\text {nd }}\right)+\mathrm{P}\left(\mathrm{D}\right.$ killed $\mathrm{A}$, and $\mathrm{C}$ dies $\left.2^{\text {nd }}\right)+\mathrm{P}\left(\mathrm{C}\right.$ killed $\mathrm{A}$ and $\left.\mathrm{C} \operatorname{dies} 2^{\text {nd }}\right)+\mathrm{P}(\mathrm{B}$ killed $\mathrm{A}$, and $\mathrm{C}$ dies $\left.2^{\text {nd }}\right)+\mathrm{P}\left(\mathrm{A}\right.$ killed $\mathrm{B}$, and $\mathrm{C}$ dies $\left.2^{\text {nd }}\right)$. Or, using our notation and the formulas derived above,

$$
\begin{aligned}
P_{32}=\frac{e}{1-\overline{\mathrm{e}} \overline{\mathrm{d}} \overline{\mathrm{b}} \bar{c} \overline{\mathrm{a}}} * \frac{\bar{d} \bar{c} b}{1-\bar{e} \bar{d} \bar{c} \bar{b}}+\frac{\bar{e} d}{1-\bar{e} \bar{d} \bar{c} \bar{b} \bar{a}} * \frac{\bar{c} b}{1-\bar{e} \bar{d} \bar{c} \bar{b}}+\frac{\bar{e} \bar{d} c}{1-\bar{e} \bar{d} \bar{c} \bar{b} \bar{a}} * \frac{b}{1-\bar{e} \bar{d} \bar{c} \bar{b}}+\frac{\bar{e} \bar{d} \bar{c} b}{1-\bar{e} \bar{d} \bar{c} \bar{b} \bar{a}} * \frac{\bar{e} \bar{d} \bar{c} b}{1-\bar{e} \bar{d} \bar{c} \bar{b}} \\
+\left(\frac{\bar{e} \bar{d} \bar{c} a}{1-\bar{e} \bar{d} \bar{c} \bar{a}}\right) *\left(\frac{\bar{e} \bar{d} \bar{c} \bar{b} a}{1-\bar{e} \bar{d} \bar{c} \bar{b} \bar{a}}\right)
\end{aligned}
$$

$\mathrm{P}\left(\mathrm{B}\right.$ dies $\left.2^{\text {nd }}\right)=\mathrm{P}\left(\mathrm{E}\right.$ killed $\mathrm{A}$, and $\mathrm{B}$ dies $\left.2^{\text {nd }}\right)+\mathrm{P}\left(\mathrm{D}\right.$ killed $\mathrm{A}$, and $\mathrm{B}$ dies $\left.2^{\text {nd }}\right)+\mathrm{P}\left(\mathrm{C}\right.$ killed $\mathrm{A}$ and $\left.\mathrm{B} \operatorname{dies} 2^{\text {nd }}\right)+\mathrm{P}(\mathrm{B}$ killed $\mathrm{A}$, and $\mathrm{B}$ dies $2^{\text {nd }}$ )

$$
\begin{aligned}
P_{22}=\frac{e}{1-\bar{e} \bar{d} \bar{b} \bar{c} \bar{a}} & \frac{d+\bar{d} c+\bar{d} \bar{c} \bar{b} e}{1-\bar{e} \bar{d} \bar{c} \bar{b}}+\frac{\bar{e} d}{1-\bar{e} \bar{d} \bar{c} \bar{b} \bar{a}} * \frac{c+\bar{c} \bar{b} e+\bar{c} \bar{b} \bar{e} d}{1-\bar{e} \bar{d} \bar{c} \bar{b}}+\frac{\bar{e} \bar{d} c}{1-\bar{e} \bar{d} \bar{c} \bar{b} \bar{a}} * \frac{\bar{b} e+\bar{b} \bar{e} d+\bar{b} \bar{e} \bar{d} c}{1-\bar{e} \bar{d} \bar{c} \bar{b}}+\frac{\bar{e} \bar{d} \bar{c} b}{1-\bar{e} \bar{d} \bar{c} \bar{b} \bar{a}} \\
& * \frac{e+\bar{e} d+\bar{e} \bar{d} c}{1-\bar{e} \bar{d} \bar{c} \bar{b}}
\end{aligned}
$$

We have already calculated the probability of A dying $2^{\text {nd }}$. We rewrite it here for the sake of completion.

$$
P_{12}=\left(\frac{e+\bar{e} d+\bar{e} \bar{d} c}{1-\bar{e} \bar{d} \bar{c} \bar{a}}\right) *\left(\frac{\bar{e} \bar{d} \bar{c} \bar{b} a}{1-\bar{e} \bar{d} \bar{c} \bar{b} \bar{a}}\right)
$$

So, we now have the probability of all the $2^{\text {nd }}$ deaths.

The reader now has an idea about how to proceed for the $3^{\text {rd }}$ and $4^{\text {th }}$ deaths. Since the formulas for B,C,D, and E dying $3^{\text {rd }}$ or $4^{\text {th }}$ become very tedious, we will not write them in this paper but have placed them in a google document and so any interested reader can find them in Agrawal (2020). However, the formulas for the elimination of A are not that long, and we will continue with them in this paper.

\section{3. $A$ is the $3^{\text {rd }}$ or $4^{\text {th }}$ one to be Eliminated}

$\mathrm{P}\left(\mathrm{A}\right.$ dies $\left.3^{\text {rd }}\right)=\mathrm{P}\left(\mathrm{A}\right.$ survives the $1^{\text {st }}$ and $2^{\text {nd }}$ deaths, and A gets killed $\left.3^{\text {rd }}\right)$

Note that since A has survived the $1^{\text {st }}$ death, so A eliminated B, and since A has survived the $2^{\text {nd }}$ death, so A has eliminated C. Also, either E or D will now eliminate A. Thus: 


$$
P_{13}=\left(\frac{\bar{e} \bar{d} \bar{c} a}{1-\bar{e} \bar{d} \bar{c} \bar{a}}\right) *\left(\frac{\bar{e} \bar{d} \bar{c} \bar{b} a}{1-\bar{e} \bar{d} \bar{c} \bar{b} \bar{a}}\right) * \frac{e+\bar{e} d}{1-\bar{e} \bar{d} \bar{a}}
$$

Where the terms in parentheses give the probability that A eliminated $\mathrm{C}$, and the remaining expression is the probability of $\mathrm{E}$ or D eliminating $\mathrm{A}$ as the $3^{\text {rd }}$ one to be eliminated.

$\mathrm{P}\left(\right.$ A dies $\left.4^{\text {th }}\right)=\mathrm{P}\left(\right.$ A survives the $1^{\text {st }}, 2^{\text {nd }}$, and $3^{\text {rd }}$ deaths, and A gets killed $\left.4^{\text {th }}\right)$

Note that since A has survived the $1^{\text {st }}$ death so A eliminated B, and since A has survived the $2^{\text {nd }}$ death so A has eliminated $\mathrm{C}$, and since A has survived the $3^{\text {rd }}$ death, so A has eliminated D. This means that now E will eliminate A. Thus:

$$
P_{14}=\left(\frac{\bar{e} \bar{d} \bar{c} \bar{b} a}{1-\bar{e} \bar{d} \bar{c} \bar{b} \bar{a}}\right) *\left(\frac{\bar{e} \bar{d} \bar{c} a}{1-\bar{e} \bar{d} \bar{c} \bar{a}}\right) *\left(\frac{\bar{e} \bar{d} a}{1-\bar{e} \bar{d} \bar{a}}\right) * \frac{e}{1-\bar{e} \bar{a}}
$$

Where the terms in parentheses give the probability that A eliminated $\mathrm{D}$, and the remaining expression is the probability of E eliminating A.

\section{Winning Probability of A}

$\mathrm{P}(\mathrm{A}$ wins $)=1-\mathrm{P}\left(\mathrm{A}\right.$ is the $1^{\text {st }}, 2^{\text {nd }}, 3^{\text {rd }}$, or $4^{\text {th }}$ one to be eliminated $)$

$$
\begin{gathered}
=1-P_{11}-P_{12}-P_{13}-P_{14} \\
=1-\frac{e+\bar{e} d+\bar{e} \bar{d} c+\bar{e} \bar{d} \bar{c} b}{1-\bar{e} \bar{d} \bar{c} \bar{b} \bar{a}}-\left(\frac{e+\bar{e} d+\bar{e} \bar{d} c}{1-\bar{e} \bar{d} \bar{c} \bar{a}}\right) *\left(\frac{\bar{e} \bar{d} \bar{c} \bar{b} a}{1-\bar{e} \bar{d} \bar{c} \bar{b} \bar{a}}\right)-\left(\frac{\bar{e} \bar{d} \bar{c} a}{1-\bar{e} \bar{d} \bar{c} \bar{a}}\right) *\left(\frac{\bar{e} \bar{d} \bar{c} \bar{b} a}{1-\bar{e} \bar{d} \bar{c} \bar{b} \bar{a}}\right) * \frac{e+\bar{e} d}{1-\bar{e} \bar{d} \bar{a}} \\
-\left(\frac{\bar{e} \bar{d} \bar{c} \bar{b} a}{1-\bar{e} \bar{d} \bar{c} \bar{b} \bar{a}}\right) *\left(\frac{\bar{e} \bar{d} \bar{c} a}{1-\bar{e} \bar{d} \bar{c} \bar{a}}\right) *\left(\frac{\bar{e} \bar{d} a}{1-\bar{e} \bar{d} \bar{a}}\right) * \frac{e}{1-\bar{e} \bar{a}}
\end{gathered}
$$

The following table provides the winning probabilities of A for several marksmanship probabilities.

Table 1. Winning Probabilities of A for some Plausible Values of Marksmanship Abilities

\begin{tabular}{|l|l|l|l|l|l|l|l|l|l|l|}
\hline $\mathrm{a}$ & $\mathrm{b}$ & $\mathrm{c}$ & $\mathrm{d}$ & $\mathrm{e}$ & $\mathrm{P}_{11}$ & $\mathrm{P}_{12}$ & $\mathrm{P}_{13}$ & $\mathrm{P}_{14}$ & $\mathrm{P}_{11}+\mathrm{P}_{12}+\mathrm{P}_{13}+\mathrm{P}_{14}$ & $\mathrm{P}$ (A wins) \\
\hline 1 & .9 & .8 & .7 & .6 & .9976 & .00234 & .00005 & .00005 & 1.0000 & 0 \\
\hline 1 & .7 & .5 & .3 & .1 & .9055 & .06473 & .01101 & .00188 & .98312 & .01688 \\
\hline 1 & .5 & .4 & .3 & .2 & .832 & .11155 & .02483 & .00632 & .9747 & .0253 \\
\hline 1 & .4 & .3 & .2 & .1 & .6976 & .14999 & .04267 & .01097 & .90123 & .09877 \\
\hline .9 & .8 & .7 & .6 & .5 & .98919 & .01023 & .00048 & .00005 & .99942 & .00058 \\
\hline .7 & .6 & .5 & .3 & .2 & .91887 & .06377 & .00918 & .00108 & .9929 & .0071 \\
\hline .5 & .45 & .4 & .35 & .3 & .91883 & .06834 & .00905 & .00061 & .99683 & .00317 \\
\hline .5 & .3 & .2 & .1 & .05 & .68525 & .15116 & .04143 & .00329 & .88113 & .11887 \\
\hline .3 & .25 & .2 & .15 & .1 & .79711 & .13772 & .03297 & .00146 & .96926 & .03074 \\
\hline .1 & .09 & .08 & .07 & .06 & .78557 & .15198 & .03684 & .0006 & .97499 & .02501 \\
\hline .1 & .05 & .045 & .04 & .035 & .65494 & .20396 & .06633 & .00115 & .92638 & .07362 \\
\hline
\end{tabular}

It is quite remarkable that in all these cases, which seem to be decently varied, the probability of A winning is less than $10 \%$. Any player with winning probability less than $20 \%$ cannot be the most likely one to win the game, so it is reasonable to believe that A will very often not be the winner.

\section{Discussion and Conclusions}

We found a reasonable number of plausible situations that defy our null hypothesis and hence we can claim that a player's shooting ability is not directly proportional to their winning probability. A was the strongest shot and yet for several diverse and quite reasonable values of the marksmanship abilities, A always had less than $10 \%$ probability of winning the game. This is less than $20 \%$ (by far, by the way). A was never the most likely player to win the game. This shows that in a scenario of perfect competition, survival of the fittest will be unlikely. This idea could apply even to more common real-world situations if everyone agreed to target the strongest competitor in the field by teaming up against them. The point is that practically speaking, strength becomes weakness in the sense that everyone is after the skin of the strongest.

Moving ahead, we plan to give a rigorous proof of the assumption made about the shooting strategy employed by the players. In order to achieve this, we will probably need to work on a game of quadruals in the hope that those results will provide the necessary steps needed to justify this strategy in pentuals. This should help because the strategy is already known in case of truels. The study of quadruels might also be interesting because it would be the first time that 
a study would be done involving a composite number of competitors. Until now people have studied duels and truels, and this paper talks about pentuals.

Another interesting study would be to allow abstentions and to find the conditions under which abstention is a better strategy than firing. Unlike truels, where the abstention can make sense only for the weakest player, it is very likely that in case of pentuals, abstention makes sense for multiple players. However, as mentioned above it would probably be best to first do a fair amount of work on quadruels before visiting pentuals again.

\section{References}

Agrawal, Y.

$(2020 b$

August

18).

Pentual

Formulas. https://docs.google.com/document/d/e/2PACX-1vRwHwKXBjuYH6LgTfrb5pshf8aO29_cnYNmek_s5JiOjmPAW H1cDoEP0Avy6yHlexRD9Dhj8splCOJ4/pub

Amengual, P., \& Toral, R. (2006). Truels, or Survival of the Weakest. Computing in Science \& Engineering, 8(5), 88-95. https://doi: 10.1109/mcse.2006.99

Hare, D. R., \& Kaleem, F. (2019) Expected Winning Probabilities in Sequential Truels Under Uniform Distributions. International Journal of Statistics and Probability, 8(6), 82.

Kilgour, D. M. (1971). The Simultaneous Truel. International Journal of Game Theory, 1(1), 229-42. https://doi: $10.1007 / \mathrm{bf0} 01753447$

Kilgour, D. M. (1975). The Sequential Truel. International Journal of Game Theory, 4(3), 151-174. https://doi: $10.1007 / \mathrm{bf} 01780632$

Kilgour, D. M., \& Brams, S. J. (1997). The Truel. Mathematics Magazine, 70(5), 315. https://doi: 10.2307/2691167

Shubik, M. (1975). Game Theory and Related Approaches to Social Behavior Selections. Huntington: Krieger.

\section{Copyrights}

Copyright for this article is retained by the author(s), with first publication rights granted to the journal.

This is an open-access article distributed under the terms and conditions of the Creative Commons Attribution license (http://creativecommons.org/licenses/by/4.0/). 